\title{
Non-Alcoholic Fatty Liver Disease (NAFLD) Is it an Emerging Risk Factor for Coronary Artery Disease?
}

Non-alcoholic fatty liver disease (NAFLD) is being recognized as the most common cause of abnormal liver enzymes in the last few decades. It includes a spectrum of liver disorders ranging from simple steatosis to steatohepatitis, fibrosing steatohepatitis, and cirrhosis, which may progress to hepatocellular carcinoma; however, the most common cause of death in patients with NAFLD is coronary artery disease (CAD), not chronic liver disease. ${ }^{1}$

The prevalence of NAFLD is more common in type 2 diabetic patients $(70-80 \%$ in comparison to $20-30 \%$ in the general population), who are also at higher risk of developing advanced fibrosis and cirrhosis. ${ }^{2}$ NAFLD is regarded as the hepatic manifestation of the metabolic syndrome, since as many as $90 \%$ of NAFLD cases have at least one component of the metabolic syndrome and as many as $33 \%$ have three or more components. ${ }^{3}$ It is related with more severe insulin resistance and hypoadiponectinemia in the patients of metabolic syndrome. ${ }^{4}$ It may respond to treatments originally developed for other insulin-resistant states (e.g. diabetes mellitus type 2) such as weight loss, metformin and thiazolidinediones. ${ }^{5}$

The relationship of NAFLD with CAD is independent of classical risk factors and is only partly explained by occurrence of metabolic syndrome. ${ }^{6,7}$ In patients with clinical indications for coronary angiogram, fatty liver is associated with coronary artery disease independently of other metabolic factors. ${ }^{8}$ NAFLD has been associated with an abnormal coronary flow reserve (CFR), which is widely used to examine the integrity of coronary microvascular circulation and liver fibrosis scores are found to be an independent predictor of depressed CFR. ${ }^{9}$ NAFLD has been considered a novel risk factor for vulnerable coronary plaques,,$^{10}$ and has been associated with the increased score $(>100)$ of coronary artery calcification. ${ }^{11}$ Because of the high risk of atherosclerosis in patients with NAFLD, even without metabolic syndrome, assessment of NAFLD may be helpful for cardiovascular risk stratification. ${ }^{12}$ It might be beneficial for the NAFLD patients to undergo screening of metabolic syndrome as well as intima media thickness in order to assess the future atherosclerotic complications. ${ }^{13}$ NAFLD could not merely be a marker of cardiovascular disease (CVD), but may also be actively involved in its pathogenesis, which includes a release of pro-atherogenic factors from the liver (C-reactive protein, fibrinogen, plasminogen activator inhibitor-1, IL-6 and other inflammatory cytokines), hepatic insulin resistance, subclinical inflammation and atherogenic dyslipidemia which together lead to increased oxidative stress and endothelial dysfunction, finally promoting CAD. ${ }^{1,14}$ However, more detail studies are required to understand the role played by NAFLD in the pathogenesis of coronary artery disease.

\section{${ }^{*}$ Corresponding author}

Department of Internal Medicine, Manipal College of Medical Sciences and Manipal Teaching Hospital. Phulbari-11, Pokhara, Kaski, Nepal Email address - umidshrestha@gmail.com

\author{
Umid Kumar Shrestha*a \\ ${ }^{a}$, Manipal College of Medical Sciences, Pokhara, Nepal
}

\section{Available online}

January 25th, 2012

\section{Keywords}

coronary artery disease, non alcoholic - fatty liver disease, metabolic syndrome

\section{Citation}

Shrestha UK. Non-Alcoholic Fatty Liver Disease (NAFLD). Is it an Emerging Risk Factor for Coronary Artery Disease? Journal of Advances in Internal Medicine. 2012;01(1)1-2 
When NAFLD is superimposed on classical risk factors, the risk of CAD may further increase. A multidisciplinary approach is necessary to control the cardiovascular and liver complications related with NAFLD.

NAFLD may be an emerging risk factor, independent to the classical risk factors and can be utilized as a surrogate marker to predict CAD. However, well-designed intervention studies, randomized clinical trials and long-term follow-up studies on large representative patients with NAFLD are needed to assess the development of coronary artery disease over a period of time; these studies might help to explain the temporal evolution of NAFLD, metabolic syndrome and coronary artery disease.

\section{REFERENCES}

1. Nseir W, Shalata A, Marmor A, et al. Mechanisms linking nonalcoholic Fatty liver disease with coronary artery disease. Dig Dis Sci. 2011;56:3439-49.

2. Targher G, Marra F, Marchesini G. Increased risk of cardiovascular disease in non-alcoholic fatty liver disease: causal effect or epiphenomenon? Diabetologia. 2008;51:1947-53.

3. Almeda-Valdés P, Cuevas-Ramos D, Aguilar- Salinas CA.

Metabolic syndrome and non-alcoholic fatty liver disease. Ann Hepatol. 2009;8(Suppl 1): S18-S24.

4. Wang ZL, Xia B, Shrestha U, et al. Correlation between adiponectin polymorphisms and non-alcoholic fatty liver disease with or without metabolic syndrome in Chinese population. $J$ Endocrinol Invest. 2008;31:1086-91.

5. Adams LA, Angulo P. Treatment of non-alcoholic fatty liver disease. Postgrad Med J. 2006;82:315-22.

6.Targher G, Bertolini L, Poli F, et al. Nonalcoholic fatty liver disease and risk of future cardiovascular events among type 2 diabetic patients. Diabetes. 2005;54:354-6.

7. Arslan U, Türkoğlu S, Balcioğlu S, et al. Association between nonalcoholic fatty liver disease and coronary artery disease. Coron Artery Dis. 2007;18:433-6.

8. Wong V W, Wong G L, Yip G W, et al. Coronary artery disease and cardiovascular outcomes in patients with non-alcoholic fatty liver disease. Hepatology. Published Online First 20 May 2011.
9. Yilmaz Y, Kurt R, Yonal O, et al. Coronary flow reserve is impaired in patients with nonalcoholic fatty liver disease: association with liver fibrosis. Atherosclerosis. 2010;211:182-6. 10. Akabame S, Hamaguchi M, Tomiyasu K, et al. Evaluation of vulnerable coronary plaques and non-alcoholic fatty liver disease (NAFLD) by 64-detector multislice computed tomography (MSCT). Circ J. 2008;72:618-25.

11. Chen $\mathrm{CH}$, Nien CK, Yang CC, et al. Association between nonalcoholic fatty liver disease and coronary artery calcification. Dig Dis Sci. 2010;55:1752-60.

12. Assy N, Djibre A, Farah R, et al. Presence of coronary plaques in patients with nonalcoholic fatty liver disease. Radiology. 2010;254:393-400.

13. Aygun C, Kocaman O, Sahin T, et al. Evaluation of metabolic syndrome frequency and carotid artery intima-media thickness as risk factors for atherosclerosis in patients with nonalcoholic fatty liver disease. Dig Dis Sci. 2008;53:1352-7.

14. Targher G, Chonchol M, Miele L, et al. Nonalcoholic fatty liver disease as a contributor to hypercoagulation and thrombophilia in the metabolic syndrome. Semin Thromb Hemost. 2009;35:277-87. 